\title{
Research on data fusion optimizing filtering algorithm for SOPLAT system
}

\author{
Qiao liang \\ Department of Information and \\ Communication, Harbin Engineering \\ University, Harbin, China \\ Eiylw2009@163.com
}

\author{
Mingxin Tan \\ .Department of Information Technology \\ Huazhong Normal University \\ Wu Han, China \\ tmingxin@yahoo.com.cn
}

\author{
Qiao Yao-ying \\ Department of Information \\ Engineering, Communication \\ University of China, Beijing, China \\ Qlbslw2008@126.com
}

\begin{abstract}
Abstrac: Most currently existing algorithms used in SOPLAT (single observer passive location and tracking) system usually select single parameter measurement set which concludes only a little measured information, which means that they do not consider and make full use of spatial and temporal information as well as frequency range information which can be measured synchronously by modern electronic detecting equipment. In order to overcome this drawback, a new fusion optimization filtering algorithm for SOPLAT system is presented, this algorithm can fully utilize the redundant information coming from many sensors, which includes TOA, DOA and Doppler frequency measurements, etc. The proposed method improves further the location \& tracking accuracy, reliability and defensive operational capability of the system by using data fusion technology. As a demonstration to evaluate the method described in this paper, two simulation examples are presented which indicate the effectiveness and feasibility of this filtering algorithm, and the results show that this method has better accuracy and convergence rate than conventional algorithms.

key word: passive location; data fusion; time and direction of arrival; nonlinear system, optimization
\end{abstract}

\section{INTRODUCTION}

It is well known that SOPLAT has many superiorities, such as secret receiving and high independence. So it has been widely studied and utilized in modern EW ${ }^{[1 \sim 5]}$. In [2] the authors pioneered the study of single observer passive location and tracking arithmetic utilizing bearing and radial acceleration information. A method for tracking maneuvering target by single station was proposed in [3] by following the theory of weighted least square estimation and Kalman filtering. Generally speaking, although these above mentioned algorithms have many advantages, they also suffer some limitations, the main shortcoming of which is that they only utilized a little measured information including acceleration and DOA, they did not consider and make full use of all kinds of measured information. In fact, modern electronic detecting equipment can measure and obtain kinds of parameters information including frequency range, spatial and temporal information, which can be fused and optimised to enhance location properties. This paper introduces data fusion technology into SOPLAT system and solves the key problem of SOPLAT in case of many parameter measurements sets better. Data fusion is a real time processing process to estimate precise position of the target we care. It should be pointed out that, so far, a litter research on single observer passive location \& tracking to maneuvering emitters by using multi-sensor information has been done in our country, and the corresponding technology is not very mature yet. Motivated by domestic and foreign research on SOPLAT in recent years $^{[6 \sim 10]}$, a new promising data fusion optimization algorithm which uses frequency range, spatial and temporal information from the target is developed in this paper, and corresponding computer simulations have been done. This method can break through the localization, which was expressed in only one parameter measurement set.

\section{ESTABLISHMENT OF MATHEMATICAL MODELS}

It is assumed that the location system can measure frequency range, spatial and temporal information of the emitter at same time by means of various sensors ${ }^{[11 \sim 12]}$, the parameter measurement sets used in location are $\{\beta, \varepsilon, \dot{\beta}, \Delta T O A\}$ and $\left\{\beta, \varepsilon, \dot{\beta}, f_{d}\right\}$ respectively. The former utilizes spatial and temporal parameters and it is called system I, and the later utilizes frequency range and spatial parameters and it is called system II. The state vectors of the two sets can be written respectively as:

$$
\begin{aligned}
& \mathbf{X}_{k}=\left[\begin{array}{lllllll}
x_{k} & y_{k} & z_{k} & \dot{x}_{k} & \dot{y}_{k} & \dot{z}_{k} & \Delta T_{r k}
\end{array}\right]^{T} \\
& \mathbf{X}_{k}^{\prime}=\left[\begin{array}{lllllll}
x_{k} & y_{k} & z_{k} & \dot{x}_{k} & \dot{y}_{k} & \dot{z}_{k} & 1 / f_{T}
\end{array}\right]^{T}
\end{aligned}
$$

Where $\Delta T_{r k}=T_{r}-\hat{T}_{r}, T_{r}$ denotes repeating pulse period and $f_{T}$ presents the carrier frequency. Corresponding state equations can be represented by

$$
\begin{aligned}
& \mathbf{X}_{k+1}=\Phi_{k+1, k} \mathbf{X}_{k}+\mathbf{W}_{k} \\
& \mathbf{X}_{k+1}^{\prime}=\Phi_{k+1, k} \mathbf{X}_{k}^{\prime}+\mathbf{W}_{k}
\end{aligned}
$$

Where

$$
\Phi_{k+1, k}=\left[\begin{array}{ccc}
\mathbf{I}_{3} & T \mathbf{I}_{3} & \mathbf{0}_{3 \times 1} \\
\mathbf{0}_{4 \times 3} & \mathbf{I}_{4}
\end{array}\right] \text { is called state transition }
$$

matrix . 
$\mathbf{W}_{k}=\left[\begin{array}{lllllll}\frac{T^{2}}{2} w_{x k} & \frac{T^{2}}{2} w_{y k} & \frac{T^{2}}{2} w_{z k} & T w_{x k} & T w_{y k} & T w_{z k} & 0\end{array}\right]^{T}$ denotes statistically independent Gaussian white noise, and $E\left[\mathbf{W}_{k}\right]=0$, $E\left[\mathbf{W}_{k}, \mathbf{W}_{k}{ }^{T}\right]=\mathbf{Q}_{k} \delta_{j, k}$.

If define that common motion state vector

$$
\mathbf{Y}_{k}=\left[\begin{array}{llllll}
x_{k} & y_{k} & z_{k} & \dot{x}_{k} & \dot{y}_{k} & \dot{z}_{k}
\end{array}\right]^{T},
$$

then

$$
\mathbf{X}_{k}=\left\lfloor\begin{array}{ll}
\mathbf{Y}_{k}^{T} & \Delta T_{r k}
\end{array}\right\rfloor^{T}, \mathbf{X}_{k}^{\prime}=\left\lfloor\mathbf{Y}_{k}^{T} \quad 1 / f_{T}\right\rfloor^{T}
$$$$
\text { and } \quad \mathbf{Y}_{k+1}=\Phi^{\mathbf{Y}} \mathbf{Y}_{k}+\mathbf{W}_{k}^{\mathbf{Y}}
$$

Where $\quad \Phi^{\mathbf{Y}}=\left[\begin{array}{cc}\mathbf{I}_{3} & T \mathbf{I}_{3} \\ 0 & \mathbf{I}_{3}\end{array}\right], \quad \mathbf{W}_{k}^{\mathbf{Y}}=\mathbf{W}_{k}[1: 6]$

For the parameter measurement sets $\{\beta, \varepsilon, \dot{\beta}, \Delta T O A\}$ and $\left\{\beta, \varepsilon, \dot{\beta}, f_{d}\right\}$, Measurement equations can be shown respectively by

$$
\begin{aligned}
& \left\{\begin{array}{l}
\beta_{k}=\arctan \left(\frac{x_{k}}{y_{k}}\right)+\delta_{\beta k} \\
\varepsilon_{k}=\arctan \left(\frac{z_{k}}{\sqrt{x_{k}^{2}+y_{k}^{2}}}\right)+\delta_{c k} \\
\dot{\beta}_{\mathrm{k}}=\frac{y_{k} \dot{x}_{k}-x_{k} \dot{y}_{k}}{x_{k}^{2}+y_{k}^{2}}+\delta_{\dot{\beta} k} \\
\Delta T O A_{k}=\left(r_{k}-r_{k-1}\right) / c+N \hat{T}_{r k}+N \Delta T_{r k}+\delta_{\tau k} \\
\beta_{k}=\arctan \left(\frac{x_{k}}{y_{k}}\right)+\delta_{\beta k} \\
\varepsilon_{k}=\arctan \left(\frac{z_{k}}{\sqrt{x_{k}^{2}+y_{k}^{2}}}\right)+\delta_{c k} \\
\dot{\beta}_{\mathrm{k}}=\frac{y_{k} \dot{x}_{k}-x_{k} \dot{y}_{k}+\delta_{\dot{\beta} k}}{x_{k}^{2}+y_{k}^{2}} \\
f_{d k}=-\frac{f_{T}\left(x_{k} \dot{x}_{k}+y_{k} \dot{y}_{k}+z_{k} \dot{z}_{k}\right)}{c \sqrt{x_{k}^{2}+y_{k}^{2}+z_{k}^{2}}}+\delta_{f d k}
\end{array}\right.
\end{aligned}
$$

Above two equations can be recast in the matrix form as

$$
\begin{aligned}
& \mathbf{Z}_{k}^{1}=\mathbf{h}^{1}\left(\mathbf{X}_{k}\right)+\mathbf{N}_{k}^{1} \\
& \mathbf{Z}_{k}^{2}=\mathbf{h}^{2}\left(\mathbf{X}_{k}^{\prime}\right)+\mathbf{N}_{k}^{2}
\end{aligned}
$$

\section{DESIGN AND REALIZATION OF DATA FUSION OPTIMIZATION ALGORITHM}

3.1 The implementation strategy of data fusion optimization filtering algorithm

The execution flowchart of data fusion optimization filtering algorithm, which consists of two measurement parameter sets, is shown in Fig.1

The basic principle of data fusion optimization filtering algorithm is introduced as follows:

Firstly, the values of unknown parameters at k-1 instant are added into the two systems respectively, then the motion variables of target are estimated by means of the location

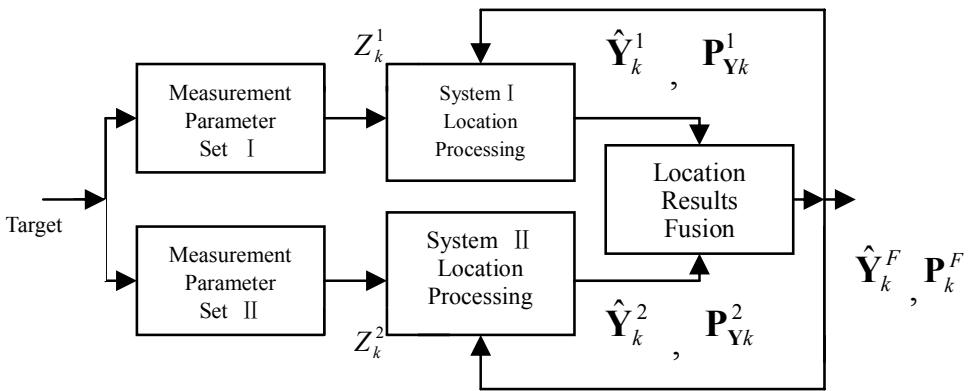

Fig.1. The flowchart of data fusion optimization filtering algorithm algorithm, consequent $\hat{\mathbf{Y}}_{k}^{1}, \hat{\mathbf{Y}}_{k}^{2}$ can be computed. Secondly, the estimated values with higher precision $\hat{\mathbf{Y}}_{k}^{F}$ may be predicted after using data fusion optimization filtering algorithm, then the fusion results $\hat{\mathbf{Y}}_{k}^{F}, \mathbf{P}_{k}^{F}$ are added into the two systems to estimate parameters $T_{r}$ and $f_{T}$. Since $\hat{\mathbf{Y}}_{k}^{F}$ obtained by data fusion optimization filtering algorithm has higher estimating precision than $\hat{\mathbf{Y}}_{k}^{1}, \hat{\mathbf{Y}}_{k}^{2}$, this is more useful to the estimation of parameters $T_{r}$ and $f_{T}$. In two systems the estimated values of $T_{r}$ and $f_{T}$ can be added and MGEKF algorithm can be used to locate and track the target.

3.2 The derivation of data fusion optimization filtering algorithm

It is assumed that at every $\mathrm{k}$ instant estimated values $\hat{\mathbf{Y}}_{k}^{1}$, $\hat{\mathbf{Y}}_{k}^{2}$ and their covariance $\mathbf{P}_{\mathbf{Y} k}^{1}, \mathbf{P}_{\mathbf{Y} k}^{2}$ on estimated error of state vector can be obtained

Let

$$
\mathbf{Z}_{k}=\left[\begin{array}{c}
\hat{\mathbf{Y}}_{k}^{1} \\
\hat{\mathbf{Y}}_{k}^{2}
\end{array}\right]=\left[\begin{array}{l}
\mathbf{I}_{6} \\
\mathbf{I}_{6}
\end{array}\right] \mathbf{Y}_{k}+\left[\begin{array}{l}
\mathbf{m}_{k}^{1} \\
\mathbf{m}_{k}^{2}
\end{array}\right] \stackrel{\Delta}{=} \mathbf{H} \mathbf{Y}_{k}+\left[\begin{array}{l}
\mathbf{m}_{k}^{1} \\
\mathbf{m}_{k}^{2}
\end{array}\right]
$$

Where $\mathbf{m}_{k}^{1}, \mathbf{m}_{k}^{2}$ are respectively errors for target motion state variables to be estimated by the two systems.

$$
\text { Let } \mathbf{M}_{k}=\left[\begin{array}{ll}
\mathbf{m}_{k}^{1} & \mathbf{m}_{k}^{2}
\end{array}\right]^{T} \text {, thus the covariance of }
$$
the errors is denoted as

$$
\mathbf{P}_{k}^{\mathbf{z}}=E\left\{\mathbf{M}_{k} \mathbf{M}_{k}^{T}\right\}=\left[\begin{array}{cc}
\mathbf{P}_{k}^{1} & \mathbf{P}_{k}^{12} \\
\mathbf{P}_{k}^{21} & \mathbf{P}_{k}^{2}
\end{array}\right]
$$

. With respect to the system $\mathrm{I}$, the filtering values at $\mathrm{k}$ instant are

$$
\hat{\mathbf{Y}}_{k \mid k}^{1}=\Phi^{\mathbf{Y}} \hat{\mathbf{Y}}_{k-1 \mid k-1}^{1}+\mathbf{K}_{k}^{1}\left[\mathbf{Z}_{k}^{1}-\mathbf{H}_{k}^{1}\left(\Phi^{\mathbf{Y}} \mathbf{Y}_{k-1 \mid k-1}^{1}\right)\right]
$$

So the estimated error can be denoted as

$$
\begin{aligned}
\tilde{\mathbf{Y}}_{k \mid k}^{1} & =\mathbf{Y}_{k}-\hat{\mathbf{Y}}_{k \mid k}^{1}=\mathbf{Y}_{k}-\hat{\mathbf{Y}}_{k \mid k-1}^{1}-\mathbf{K}_{k}^{1} \mathbf{v}_{k}^{1} \\
& =\left(\mathbf{Y}_{k}-\mathbf{Y}_{k \mid k-1}^{1}\right)-\mathbf{K}_{k}^{1}\left(\mathbf{H}_{k}^{1} \mathbf{Y}_{k}+\mathbf{N}_{k}^{1}-\mathbf{H}_{k}^{1} \hat{\mathbf{Y}}_{k \mid k-1}^{1}\right) \\
& =\left(\mathbf{I}-\mathbf{K}_{k}^{1} \mathbf{H}_{k}^{1}\right) \Phi^{\mathbf{Y}} \widetilde{\mathbf{Y}}_{k-1 \mid k-1}^{1}+\left(\mathbf{I}-\mathbf{K}_{k}^{1} \mathbf{H}_{k}^{1}\right) \mathbf{W}_{k-1}^{\mathbf{Y}}-\mathbf{K}_{k}^{1} \mathbf{N}_{k}^{1}
\end{aligned}
$$$$
\text { Where } \mathbf{v}_{k}^{1}=\mathbf{Z}_{k}^{1}-\mathbf{H}_{k}^{1}\left(\Phi^{\mathbf{Y}} \mathbf{Y}_{k-1 \mid k-1}^{1}\right) \text { is called }
$$ 
new information vector of the system at $k$ instant. And

$$
\begin{aligned}
& \tilde{\mathbf{Y}}_{k-1 \mid k-1}^{1}=\mathbf{Y}_{k-1}-\hat{\mathbf{Y}}_{k-1 \mid k-1}^{1} \\
& \mathbf{H}_{k}^{1}=\left.\frac{\partial \mathbf{h}^{1}\left(\mathbf{X}_{k}\right)}{\partial \mathbf{Y}_{k}}\right|_{\mathbf{Y}_{k}=\hat{\mathbf{Y}}_{k \mid k-1}^{1}} .
\end{aligned}
$$

For the system II we may compute and obtain $\tilde{\mathbf{Y}}_{k \mid k}^{2}$ in the same manner.

So the inter covariance is written as

$$
\begin{aligned}
\mathbf{P}_{k}^{12} & =E\left\{\widetilde{\mathbf{Y}}_{k \mid k}^{1}\left(\widetilde{\mathbf{Y}}_{k \mid k}^{2}\right)^{T}\right\} \\
& =\left(\mathbf{I}-\mathbf{K}_{k}^{1} \mathbf{H}_{k}^{1}\right)\left[\Phi^{\mathbf{Y}} \mathbf{P}_{k-1}^{12}\left(\Phi^{\mathbf{Y}}\right)^{T}\right. \\
+ & \left.\mathbf{Q}_{k-1}^{\mathbf{Y}}\right]\left(\mathbf{I}-\mathbf{K}_{k}^{2} \mathbf{H}_{k}^{2}\right)^{T}+\mathbf{K}_{k}^{1} \mathbf{R}_{k}^{12}\left(\mathbf{K}_{k}^{2}\right)^{T}
\end{aligned}
$$

Where $\mathbf{Q}_{k-1}^{\mathbf{Y}}=E\left\{\mathbf{W}_{k}^{\mathbf{Y}}\left(\mathbf{W}_{k}^{\mathbf{Y}}\right)^{T}\right\}$ is noise variance of state vector,

$$
\mathbf{R}_{k}^{12}=E\left\{\mathbf{N}_{k}^{1}\left(\mathbf{N}_{k}^{2}\right)^{T}\right\}=\left[\begin{array}{cccc}
\sigma_{\beta}^{2} & 0 & 0 & 0 \\
0 & \sigma_{\varepsilon}^{2} & 0 & 0 \\
0 & 0 & \sigma_{\dot{\beta}}^{2} & 0 \\
0 & 0 & 0 & 0
\end{array}\right]
$$

denotes noise covariance of measurement vectors of the two systems.

The fusion estimated values of target motion vector could be obtained by using weighted least square location algorithm in equation (7)

$$
\hat{\mathbf{Y}}_{k}^{F}=\left[\mathbf{H}^{T}\left(\mathbf{P}_{k}^{\mathbf{Z}}\right)^{-1} \mathbf{H}\right] \mathbf{H}^{T}\left(\mathbf{P}_{k}^{\mathbf{Z}}\right)^{-1} \mathbf{Z}_{k}
$$

Its estimated error is $\mathbf{P}_{k}^{F}=\left[\mathbf{H}\left(\mathbf{P}_{k}^{\mathbf{Z}}\right)^{-1} \mathbf{H}\right]^{-1}$

According to above process, data fusion estimated results of target motion state $\mathbf{Y}_{k}$ in two systems can be obtained, then adding $\hat{\mathbf{Y}}_{k}^{F}, \mathbf{P}_{k}^{F}$ into system I and system II respectively to further estimate target parameters $T_{r}$ and $f_{T}$.

\section{SIMULATION TESTS}

Here two experiments are designed to evaluate and account for the filter performance characteristics of the data fusion optimizing filtering algorithm. The estimation algorithm described in section III was programmed on a digital computer and subsequently tested with simulated data.

Simulation A: Consider the following engagement scenario. It is assumed that initial motion state of the emitter is $\mathbf{X}_{0}=\left[\begin{array}{llll}120 \mathrm{~km} & 80 \mathrm{~km} 8 \mathrm{~km}-300 \mathrm{~m} / \mathrm{s} 150 \mathrm{~m} / \mathrm{s} \quad 0 \mathrm{~m} / \mathrm{s}\end{array}\right]^{T}$. The observer station is on origin and is assumed to be stationary. Measurement variable sets include $\{\beta, \varepsilon, \dot{\beta}, \Delta T O A\}$ and $\left\{\beta, \varepsilon, \dot{\beta}, f_{d}\right\}$. Pulse period of the emitter $T_{r}=1 \mathrm{~ms}$, wave-length is $0.1 \mathrm{~m}$. To reduce influence of random factor, 100 times Monte-Carlo test method is used.

A realistic description of data fusion optimization filtering algorithm is provided by the data shown in fig.2. In contrast, this data fusion optimization filtering algorithm has higher convergence speed and location precision than either system I or system II, which is the most important aspect in passive location problem. The intuitive results shown in fig. 2 means that under the same circumstance performance of the system can be greatly enhanced by means of adapting data fusion optimization filtering algorithm. In fact this result can be deduced easily from the calculating process of data fusion optimization filtering algorithm.

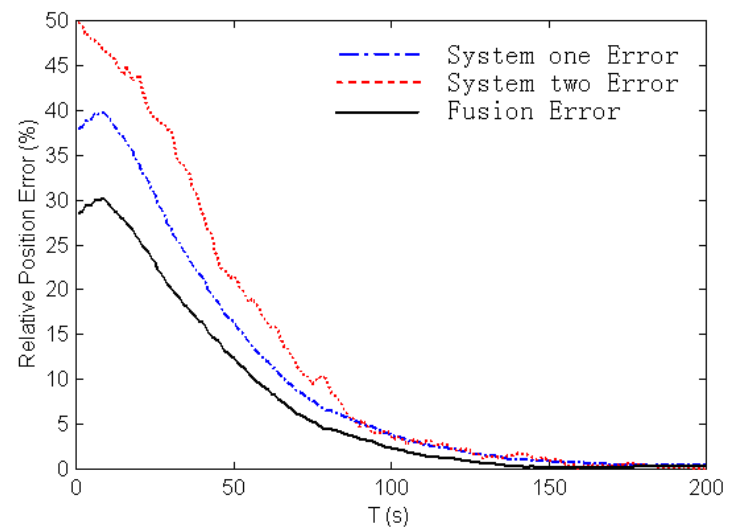

Fig.2. Simulation results of data fusion optimization filtering algorithm

Simulation B: It is assumed that simulation condition of simulation two is basically the same as simulation one. But in this simulation test the measurement error in either system I or system II is supposed to be bigger, the corresponding simulation results of data fusion optimization filtering algorithm are shown in fig. 3(a), (b).

The simulation results in fig. 3(a), (b) show respectively that when measurement error in either system I or system II is bigger to make the location function be worse, the result of data fusion optimization filtering algorithm will approach and will be superior to the better one of the two measurement variable sets in location property.

By comparison of coordinate values from figure 3(a) to figure 3(b), it can be found that when change in measurement accuracy takes place, great change in both location accuracy and convergence velocity will occur in only system I and II, but there is less influence on the proposed data fusion optimization filtering algorithm. This demonstrates that the algorithm has higher stability, which has been proved by lots of experimental results. Its filtering precision and stability excel those of either of the two measurement sets. Although numerous simulation tests were conducted during the study, only two representatives of results have been presented. Nevertheless, these results are sufficient to demonstrate the performance of the algorithm given in this work.

\section{CONCLUSION}

In this article, a novel data fusion optimization filtering algorithm is presented to effectively overcome drawbacks in the conventional algorithms. The validity of the approach has been illustrated via two important simulations. In addition, in 
the study we have compared the data fusion optimizing filtering algorithm with conventional algorithms.

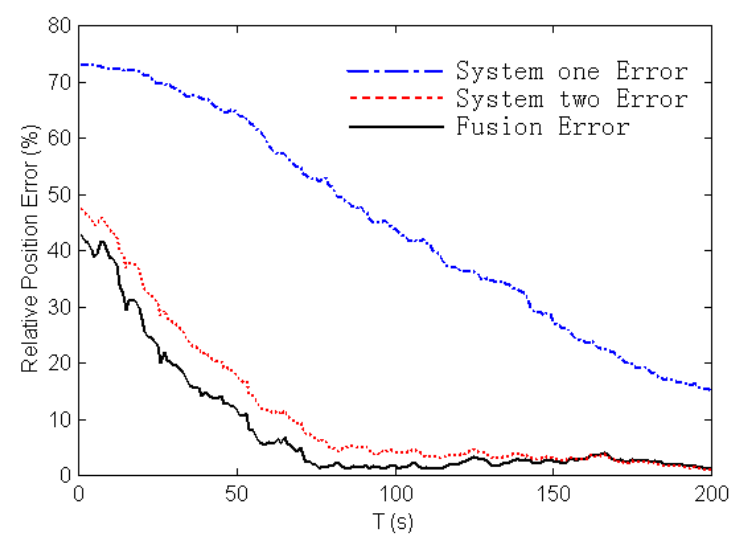

(a) The measurement error in system I is bigger

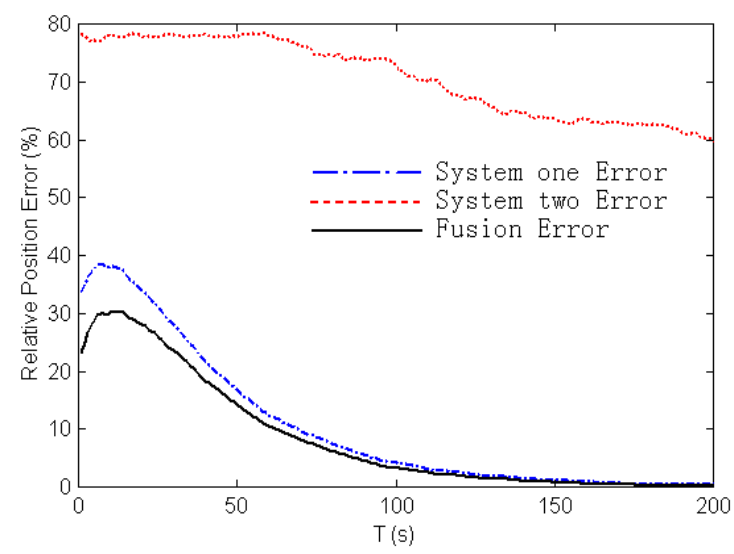

(b) The measurement error in system II is bigger,

Fig.3. Simulation results of data fusion algorithm for single passive location

The simulated results show that this new algorithm has obtained significant improvement in the estimate performance. Summarily, the data fusion optimizing filtering algorithm proposed in this paper is a valid method for nonlinear SOPLAT system. It has potential utilizations in practical military applications due to its simplicity and effectiveness. It should be stressed that, however, the assumption made in the algorithm discussed here will probably be modified according to the needs of the specific applications. The presentation of such an analysis is beyond the scope of this paper and will be discussed in future publications.

\section{REFERENCES}

[1] CHENG L M, KONG L Research on methods and application of information fusion [J]. Transducer and Microsystem Technologies, 2007 , 26 (3) : 4-9

[2] FENG D W, LI Z H. A new single observer passive location and tracking technology and its observability analysis [J]. Journal of National University of Defense Technology, 2004,26 (1) : 68-71

[3] JIAO S H, LIU S J, SI X C TDOA passive location adaptive algorithm to maneuvering target [J]. Journal of Harbin Engineering University, 2001,22 (5) : 57-63

[4] WANG H L, CHEN X X, LU Q H. A new adaptive filtering algorithm for systems with multiplicative noise [J]. Journal of Harbin Institute of Technology (New Series), 2005, 12 (1) : 71-73

[5] WANG H P, Li S W. Information fusion algorithm based on fuzzy Kalman filter [J]. Computer Measurement \& Control. 2006, 14(8) : 1230-1234

[6] ZHANG Y A, ZHOU D, DUAN G R Cramér-Rao lower bound analysis for guidance systems with bearings-only measurements [J]. Journal of Harbin Institute of Technology (New Series), 2007, 14 (2) : $229-231$

[7] XU D, LIU Y A, LIU T M Study on Passive Location and Data Fusion of the Low-altitude OTHT. Modern Radar[J], 2007, 29(8) : 22-25.

[8] LI Q L, FU Y F. Multi-sonar data fusion using Kalman filter [J]. Computer Simulation, 2007, 24(8) : 299-302.

[9] ZHENG M, ZHAO Y Q. Maneuvering target tracking based on fusion of multi-sensor [J]. Journal of Detection \& Control, 2006,28(5) : 43-45.

[10] R. K. Saha. Track-to-track fusion with dissimilar sensors [J]. IEEE Trans. on Aerospace and Electronic Systems, vol.32, no.3, 1996: 1021-1028

[11] LIU Y A, CHEN S C. Application of passive location and data fusion techniques in emitter target tracking [J]. Systems Engineering and Electronics, 2003, 25 (8) : 946-949。

[12] R. K. Saha, K. C. Chang, An efficient algorithm for multisensor track fusion [J]. IEEE Trans. on Aerospace and Electronic Systems, vol.34, no. $1,1998: 200-210$ 